\title{
Sonographic Findings of the Primary Mucoepidermoid Carcinoma of the Breast
}

\author{
Gui-Wu Chen ${ }^{1}$, Wen-Qin Liu ${ }^{1}$, Xiao-Min Liao ${ }^{1}$, Fen-Fen $\mathrm{Yu}^{1}$, and Yuhuan Xie ${ }^{1}$ \\ ${ }^{1}$ Dongguan People's Hospital
}

December 14, 2021

\section{Sonographic Findings of the Primary Mucoepidermoid Carcinoma of the Breast}

Gui-Wu Chen ${ }^{1}$, Wen-Qin Liu ${ }^{1}$, Xiao-Min Liao ${ }^{2}$, Fen-Fen $\mathrm{Yu}^{3}$, Yu-Huan Xie ${ }^{1}$

${ }^{1}$ Department of Ultrasound, Affiliated Dongguan Hospital, Southern Medical University, Dongguan, China

${ }^{2}$ Department of Pathology, Affiliated Dongguan Hospital, Southern Medical University, Dongguan, China

${ }^{3}$ Department of Radiology, Affiliated Dongguan Hospital, Southern Medical University, Dongguan, China

Running Title: Mucoepidermoid Carcinoma of the Breast

Corresponding Author:

Yu-Huan Xie

Department of Ultrasound, Dongguan People's Hospital, Southern Medical University, No.78, Wandao Road, Dongguan City, Dongguan 52300, Guangdong Province, China.

Telephone Number: +86 13713130989

Fax Number: 0769-28636378

E-mail Address: xieyuhuan0989@163.com

First Author:

Gui-Wu Chen

Department of Ultrasound, Dongguan People's Hospital, Southern Medical University, No.78, Wandao Road, Dongguan City, Dongguan 52300, Guangdong Province, China.

Telephone Number: +86 13427808564

E-mail Address: chenguiwu_6996@163.com

ORCID ID: 0000-0003-2805-9007

Data Availability Statement

The data used to support the findings of this study are available from the corresponding author upon request.

\section{Funding Statement}

No funding was received for this study.

\section{Conflicts of Interest Disclosure}


The authors declare that there is no conflict of interest.

\section{Ethics of Approval Statement and Clinical Trial Registration}

The corresponding author had the written consent of the patient to use the data for publication.

\section{Patient Consent Statement}

The patient agreed to use his/her information and samples (including blood, urine, excrement, and excised tissue) for medical research for non-commercial purposes under the premise of strict privacy protection.

\section{Permission to Reproduce Material from Other Sources}

No material from other source was applied.

Title: Sonographic Findings of the Primary Mucoepidermoid Carcinoma of the Breast

Running Title: Mucoepidermoid Carcinoma of the Breast

Keywords: mucoepidermoid carcinoma, breast tumor, mammography, ultrasonography, magnetic resonance imaging

\section{Number of Characters in the Title: 74}

Number of Words in the Manuscript: 493

\section{Number of Figures: 3}

\section{Number of Tables: 0}

Mucoepidermoid carcinoma (MEC) is an invasive tumor that has been reported in many organs, such as the salivary glands, lungs, esophagus, and thymus; however, it rarely affects the breast. ${ }^{1}$ Only a few case reports about the primary MEC of the breast are available that provide pathological evidence, which is limited to imaging, including mammography, ultrasonography, and magnetic resonance imaging.

A 38-year-old woman with a history of breast masses for 1 year presented to our hospital with a mass in the right breast, which had been rapidly grown in 1 week. On physical examination, the biggest mass was palpated in the lower outer quadrant of the right breast, which felt pliable but strong, was well-defined and movable. Before being admitted to the hospital, mammography was performed, which revealed a well-defined huge mass with a partly-lobulated boundary (Figure 1).

Initially, breast ultrasound examinations were performed during her hospital admission. Multiple cystic breast masses bilaterally were observed; most of them were oval, anechoic, well-defined, and thin-walled. However, the biggest mass was some solid tissue within the septa-divided cystic spaces with rich blood flow signals and high resistance (Figure 2). No lymph node metastasis was found in the bilateral axillary nodes.

Afterward, magnetic resonance imaging (MRI) of the breast was performed before excising the biggest mass, which showed different signals on T1-weighted and T2-weighted imaging at the solid tissue, while both revealed a high signal at the mass peripherally. Additionally, it was observed that the lateral thoracic artery of the right breast was thicker on maximum intensity projection (Figure 3).

Eventually, the patient underwent surgical resection of the biggest mass and a diagnosis of primary lowgrade MEC was confirmed. No recurrence and metastasis were reported during the 6-months follow-up postoperatively.

Most of the case reports have insufficient imaging. Only a few cases of breast MEC mammography have been reported which described an unclear mass with or without accumulation of calcific deposits. Likewise, the studies reporting ultrasound imaging also lacked detailed descriptions and only mentioned minimal details. Most ultrasound images of primary breast MEC show a complex cystic or solid mass with a rough or regular surface. ${ }^{2}$ When breast ultrasound is unavailable or inconclusive, MRI offers more diagnostic information to 
confirm the characteristics of the mass, including its components and composition; however, the evidence is still too deficient to characterize the findings.

Based on the evidence describing pathological characteristics, primary breast MECs are histologically classified into three categories - low, intermediate, and high grade. These are usually characterized by a prominent cystic component, intraductal papillary proliferation, and solid architecture, respectively. Most low or intermediate-grade primary breast MECs have an indolent behavior whereas high-grade masses could be fatal due to distant metastasis. ${ }^{3}$

In summary, exploring the imaging manifestations of primary breast MEC in detail is essential for which various imaging modalities, including mammography, ultrasonography, and MRI are available. These modalities are crucial in not only characterizing the mass but may also influence the histological grading of the mass before surgical treatment.

\section{References}

1. Sherwell-Cabello S, Maffuz-Aziz A, Ríos-Luna NP, et al. Primary mucoepidermoid carcinoma of the breast. The Breast Journal , 2017;23:753-755.

2. Ye RP, Liao YH, Xia T, et al. Breast mucoepidermoid carcinoma: a case report and review of literature. International Journal of Clinical and Experimental Pathology, 2020;13:3192-3199.

3. WHO classification of tumours editorial board. breast tumors. Lyon: IARC Press, 2019;2:149-150. 


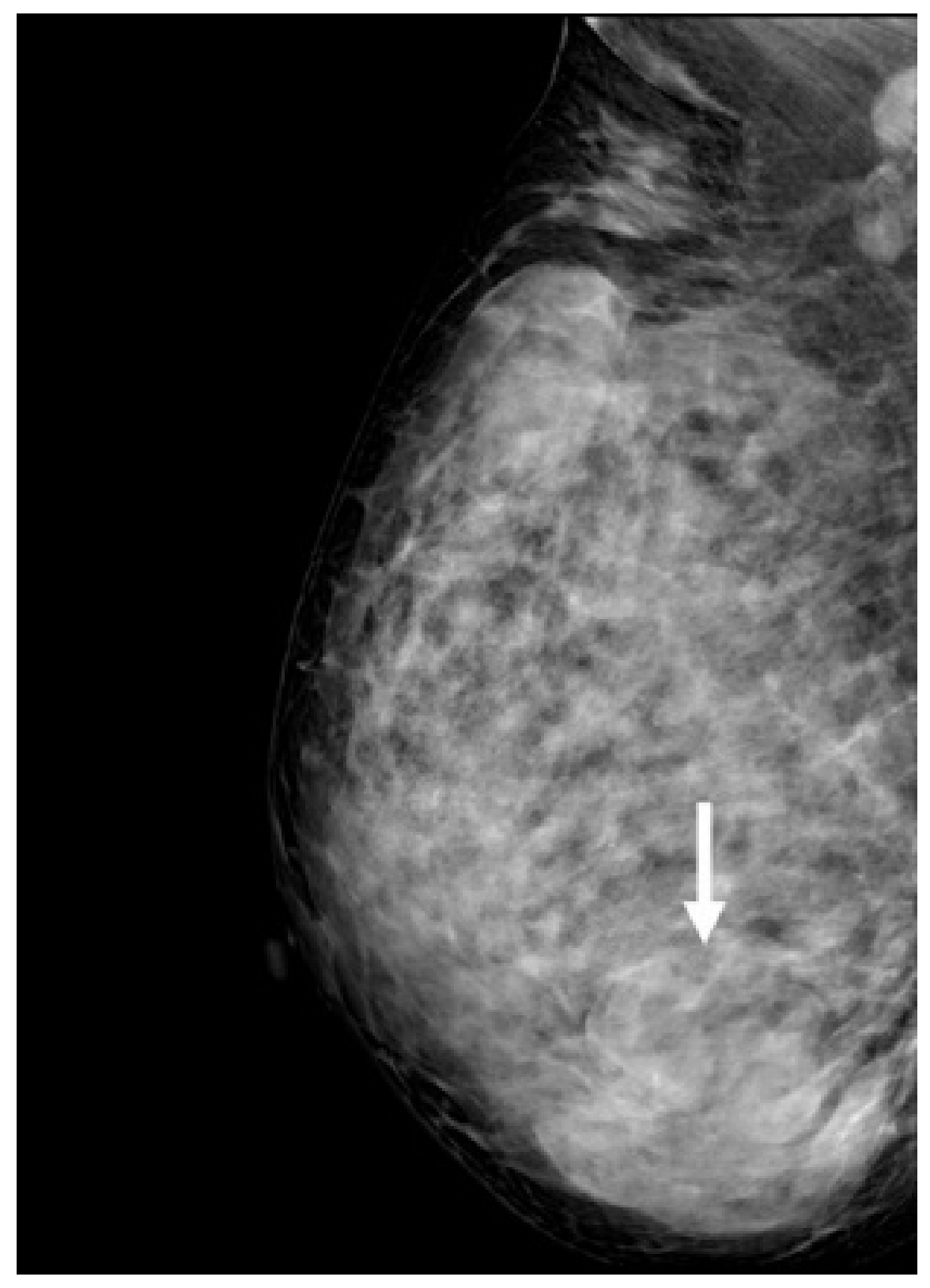




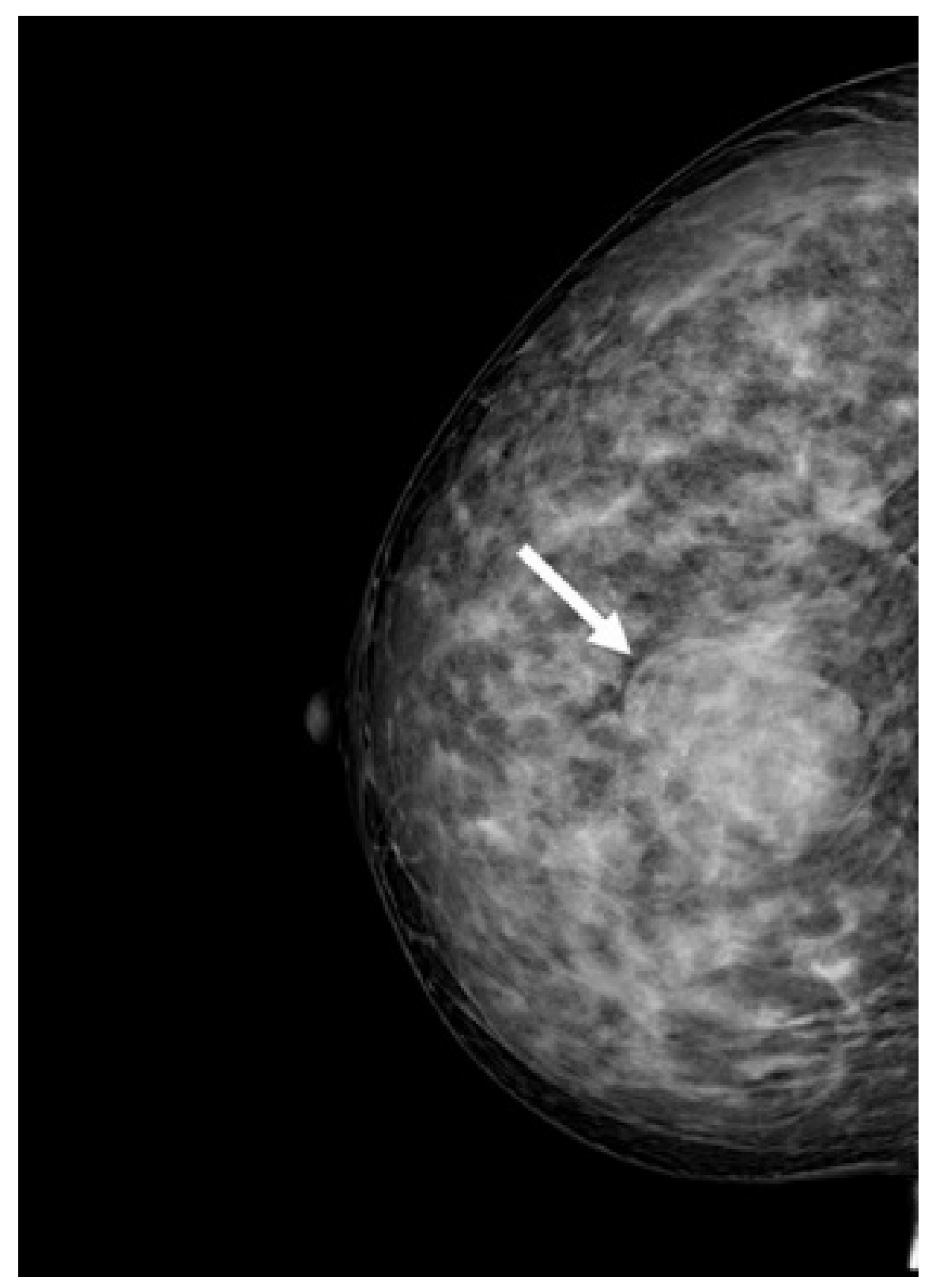

Figure 1: Mammography in the case of primary breast mucoepidermoid carcinoma showed the biggest mass was a well-defined mass with a partly-lobulated boundary as seen on the axial and coronal plane views.

\section{Hosted file}

image3.emf available at https://authorea.com/users/451161/articles/549387-sonographicfindings-of-the-primary-mucoepidermoid-carcinoma-of-the-breast 

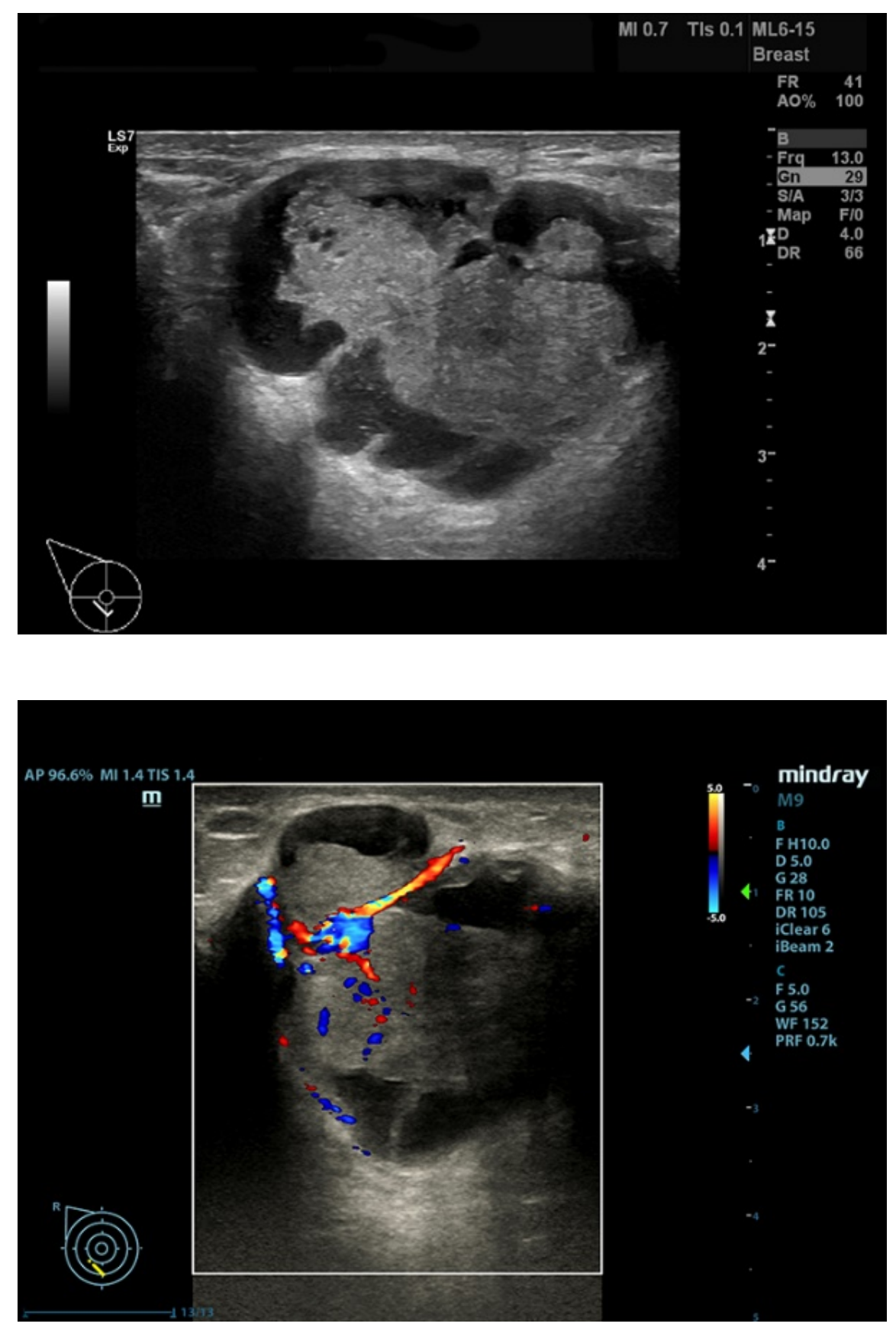


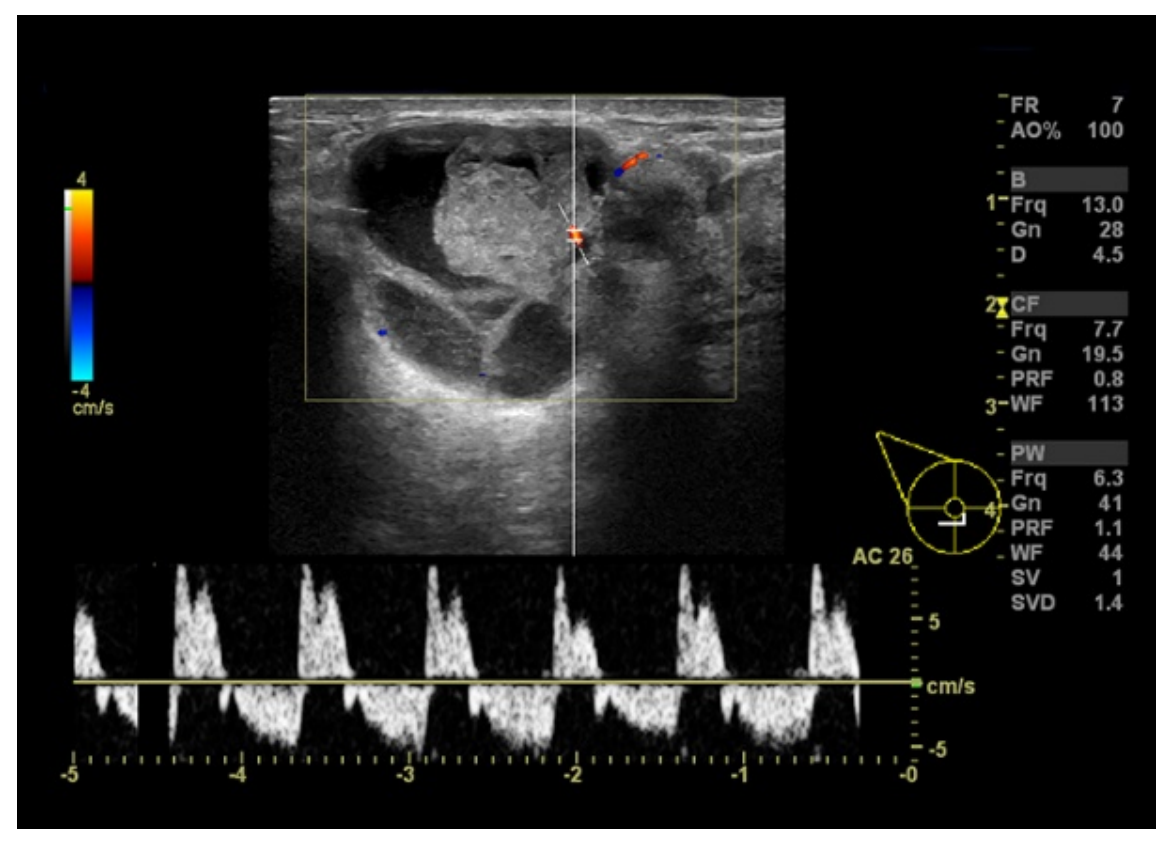

Figure 2 : Ultrasonography of primary breast mucoepidermoid carcinoma. (A) Gray-scale ultrasound showed the mass was irregular, lobulated, and well-defined. (B) Color Doppler ultrasound showed the solid tissue with rich blood flow signals. (C) Pulsed-wave Doppler ultrasound showed the blood flow had high resistance. 


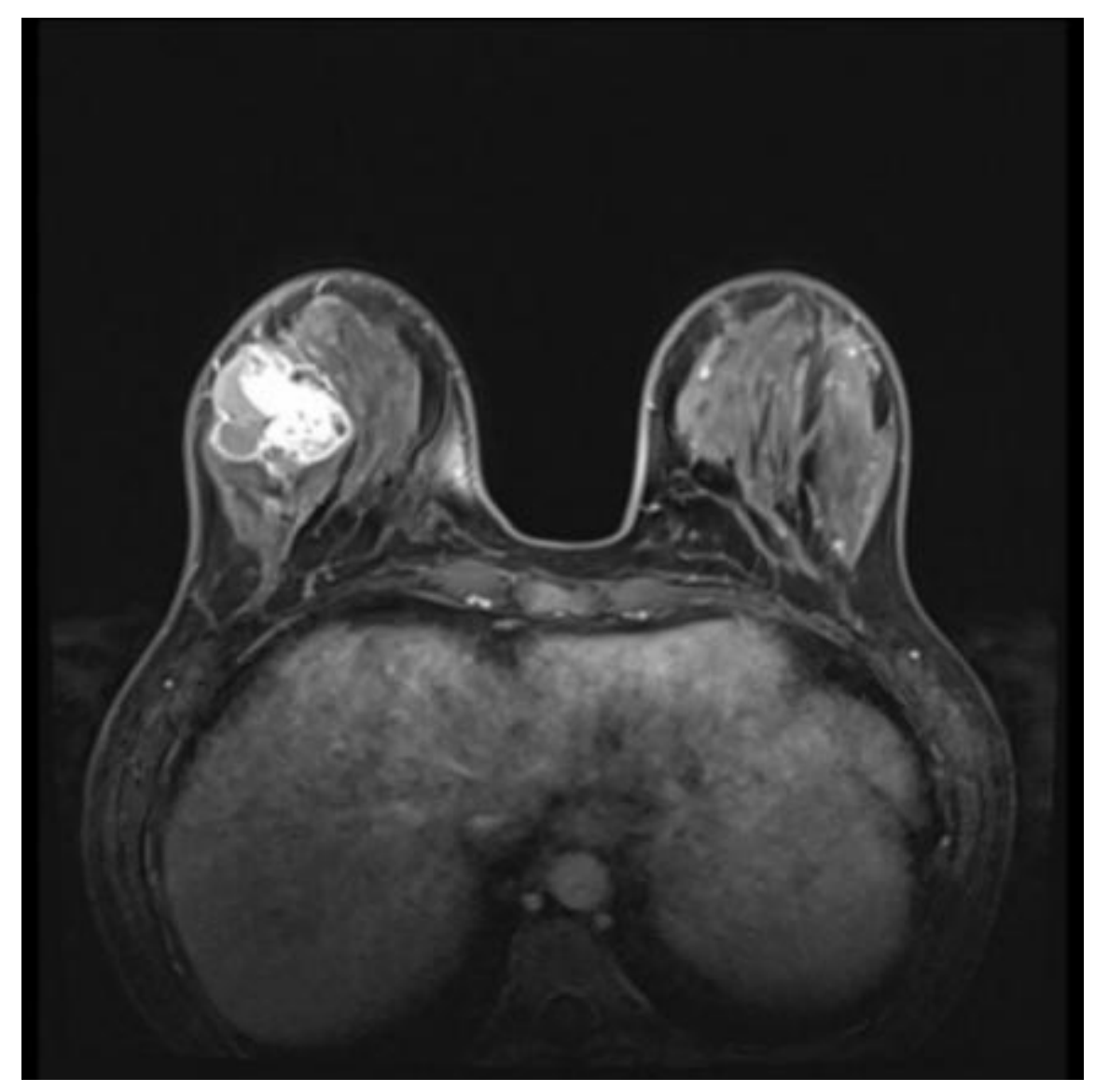




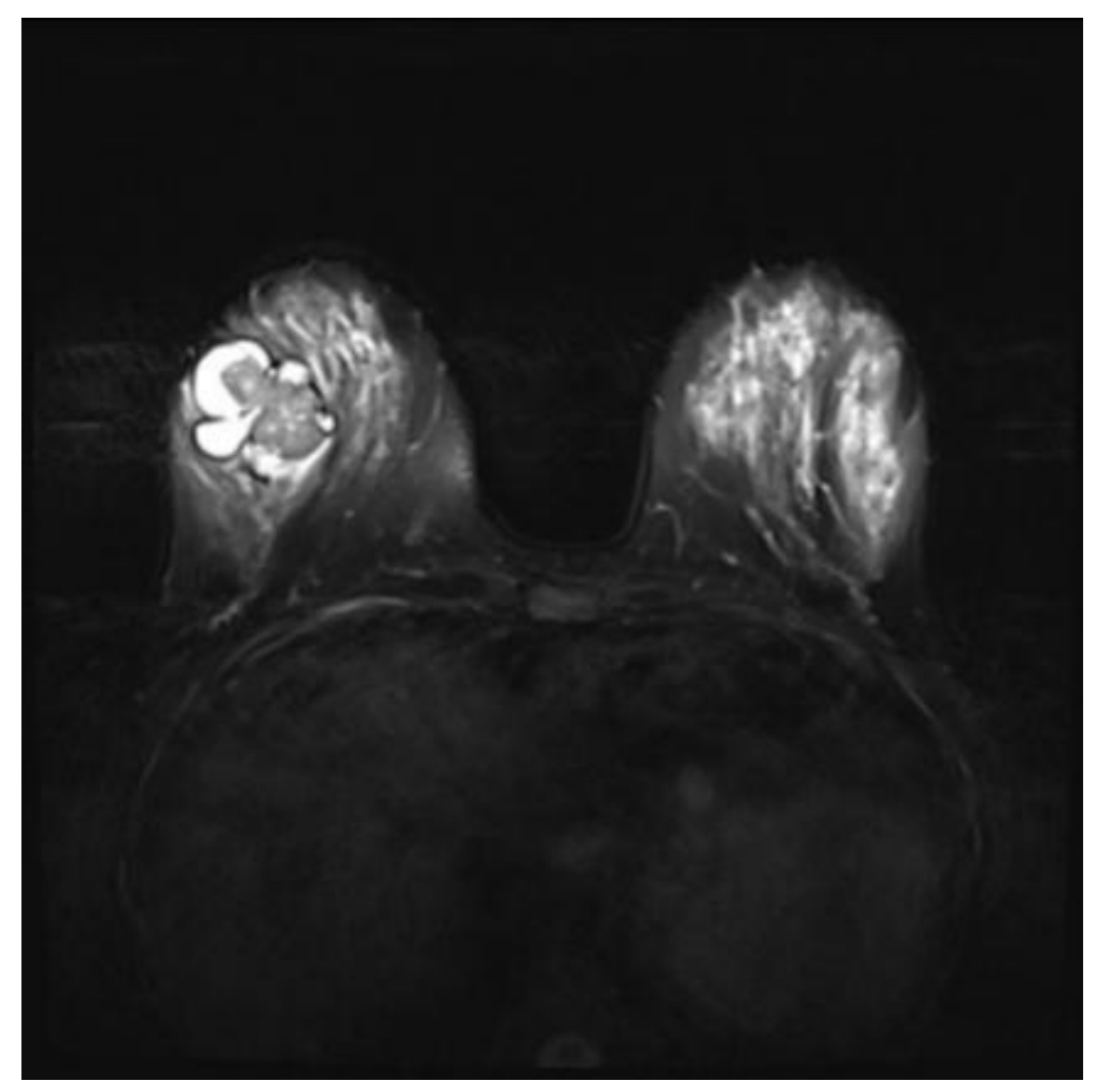




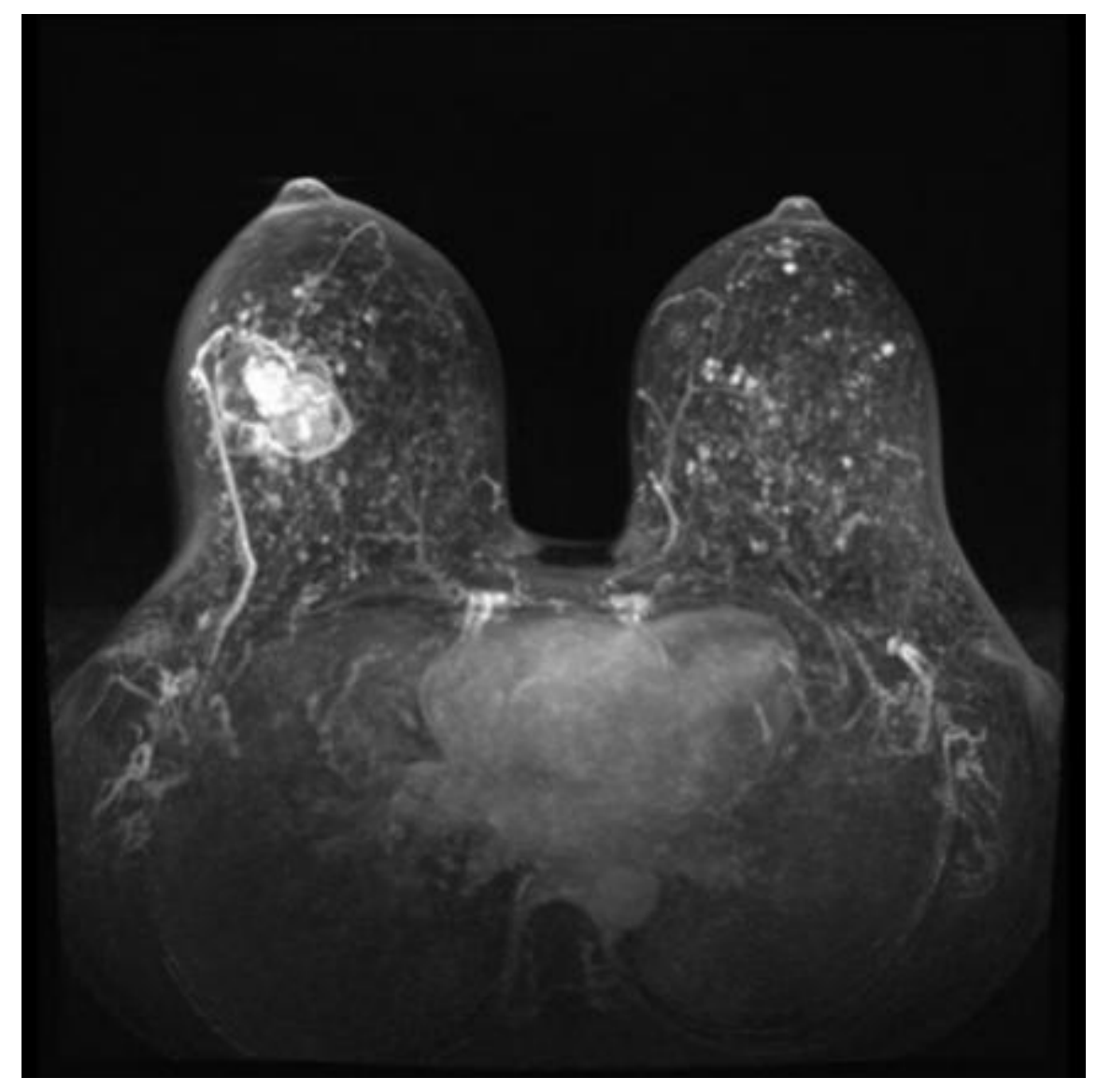

Figure 3: Magnetic resonance imaging of primary breast mucoepidermoid carcinoma. (A) T1-weighted imaging showed the solid tissue had an equal-intensity signal with a high-intensity signal at the periphery of the mass. (B) T2-weighted imaging showed the solid tissue had a low-intensity signal with a high signal at the periphery of the mass. (C) Maximum intensity projection showed the lateral thoracic artery of the right breast was thicker than the left breast. 